Therefore, the challenge is to reconstruct the CRB-65 score into a tool more valuable for this population, providing not only good prediction of the risk of death from CAP but also of the risk of hospitalisation in terms of functional deterioration. The decision to hospitalise will then be made based on this tool and on the background of the quality of onsite facilities for pneumonia treatment ${ }^{917}$ and possible DNR decisions. ${ }^{20}$

Another important issue to consider in the assessment of pneumonia severity is the incorporation of new biomarkers in refining prediction rules. ${ }^{21}$ Several biomarkers are being evaluated in CAP, including procalcitonin (PCT), ${ }^{22}{ }^{23}$ proadrenomedul$\operatorname{lin}^{24}$ and copeptin. ${ }^{25}$ All of these biomarkers have performed well. However, what is the real progress in determining biomarkers? Can they replace clinical rules or even overrule clinical judgement? Most recently, the CAPNETZ investigators demonstrated that the CRB-65 score and PCT were equally predictive of inhospital death of CAP but obviously both predictors did not measure the same. Using $0.228 \mathrm{ng} / \mathrm{ml}$ as the cut-off, deaths were highly more probable in those patients with PCT values above the cut-offs across all CRB-65 risk classes. ${ }^{23}$ If these data were validated and supported in a large population, this would be an ideal strategy to increase the amount of candidates for ambulatory treatment with very low risk of death despite elevated CRB-65 scores. At the same time, it would be a great paradigm of how basic clinical information can be refined by highly sophisticated but rapidly available laboratory investigations.

If we look back, impressive progress has been made in severity assessment of CAP, and CRB-65 seems to be a near ideal tool to help the clinician to validate his clinical judgment. If we look forward, important challenges have to be faced. These include a thorough refinement of CRB-65 by incorporation of functional parameters and reconstruction of CRB-65 for the very elderly and severely disabled population in order not only to assess the risk of death but also to prevent potential harm from hospitalisation. On the other hand, attempts to incorporate biomarkers into clinical prediction rule based algorithms for the site of care seem promising. Who could ask for more?

Competing interests: None.

Thorax 2008;63:665-666. doi:10.1136/thx.2008.099028

\section{REFERENCES}

1. Fine MJ, Auble TE, Yealy DM, et al. A prediction rule to identify low-risk patients with community-acquired pneumonia. N Engl J Med 1997;336:243-50.

2. Lim WS, Lewis S, Macfarlane JT. Severity prediction rules in community acquired pneumonia: a validation study. Thorax 2000;55:219-23.

3. Lim WS, van der Eerden MM, Laing $R$, et al. Defining community acquired pneumonia severity on presentation to hospital: an international derivation and validation study. Thorax 2003;58:377-82.

4. Ewig S, de Roux A, Bauer T, et al. Validation of predictive rules and indices of severity for community acquired pneumonia. Thorax 2004;59:421-7.

5. Bauer TT, Ewig S, Marre R, et al. The CAPNETZ Study Group: CRB-65 predicts death from communityacquired pneumonia. J Intern Med 2006;260:93-101.

6. Capelastegui A, España PP, Quintana JM, et al. Validation of a predictive rule for the management of community-acquired pneumonia. Eur Respir $J$ 2006:27:151-7.

7. Ewig S, Torres A, Woodhead M. Assessment of pneumonia severity: a European perspective. Eur Respir J 2006;27:6-8.

8. Chalmers JD, Singanayagam A, Hill AT. Systolic blood pressure is superior to other haemodynamic predictors of outcome in community acquired pneumonia. Thorax 2008;63:698-702.

9. Leff B, Burton L, Mader SL, et al. Hospital at home: feasibility and outcomes of a program to provide hospital-level care at home for acutely ill older patients. Ann Intern Med 2005;143:798-808.

10. Torres $\mathbf{O H}$, Muñoz J, Ruiz D, et al. Outcome predictors of pneumonia in elderly patients: importance of functional assessment. J Am Geriatr Soc 2004;52:1603-9.

11. Inouye SK, Peduzzi PN, Robison JT, et al. Importance of functional measures in predicting mortality among older hospitalized patients. JAMA 1998;279:1187-93.
12. Sager MA, Rudberg MA, Jalaluddin M, et al. Hospital admission risk profile (HARP): identifying older patients at risk for functional decline following acute medical illness and hospitalization. J Am Geriatr Soc 1996;:44:251-7.

13. El Solh A, Pineda $L$, Bouquin $P$, et al. Determinants of short and long term functional recovery after hospitalization for community-acquired pneumonia in the elderly: role of inflammatory markers. BMC Geriatr 2006;6:12.

14. Mody L, Sun R, Bradley SF. Assessment of pneumonia in older adults: effect of functional status. J Am Geriatr Soc 2006;54:1062-7.

15. Kruse RL, Mehr DR, Boles KE, et al. Does hospitalization impact survival after lower respiratory infection in nursing home residents? Med Care 2004; 42:860-70.

16. Fried TR, Gillick MR, Lipsitz LA. Whether to transfer? Factors associated with hospitalization and outcome of elderly long-term care patients with pneumonia. J Gen Intern Med 1995;10:246-50.

17. Loeb M, Carusone SC, Goeree R, et al. Effect of a clinical pathway to reduce hospitalizations in nursing home residents with pneumonia: a randomized controlled trial. JAMA 2006:295:2503-10.

18. Fried TR, Gillick MR, Lipsitz LA. Short-term functional outcomes of long-term care residents with pneumonia treated with and without hospital transfer. J Am Geriatr Soc 1997;45:302-6.

19. Degelau J, Guay D, Straub K, et al. Effectiveness of oral antibiotic treatment in nursing home-acquired pneumonia. J Am Geriatr Soc 1995:43:245-51

20. Marrie TJ, Fine MJ, Kapoor WN, et al. Communityacquired pneumonia and do not resuscitate orders. J Am Geriatr Soc 2002;50:290-9.

21. Christ-Crain $\mathbf{M}$, Müller B. Biomarkers in respiratory tract infections: diagnostic guides to antibiotic prescription, prognostic markers and mediators. Eur Respir J 2007;30:556-73.

22. Christ-Crain $\mathbf{M}$, Stolz D, Bingisser R, et al Procalcitonin guidance of antibiotic therapy in community-acquired pneumonia: a randomized trial. Am J Respir Crit Care Med 2006;174:84-93.

23. Krüger S, Ewig S, Marre R, et al on behalf of the CAPNETZ study group. Procalcitonin predicts patients at low risk of death from community-acquired pneumonia across all CRB-65 classes. Eur Respir J 2008:31:349-55

24. Christ-Crain M, Morgenthaler NG, Stolz D, et al. Proadrenomedullin to predict severity and outcome in community-acquired pneumonia. Crit Care 2006;10:R96

25. Krüger S, Papassotiriou J, Marre R, et al. CAPNETZ Study Group. Pro-atrial natriuretic peptide and provasopressin to predict severity and prognosis in community-acquired pneumonia: results from the German competence network CAPNETZ. Intensive Care Med 2007;33:2069-78.

\title{
The place of varenicline in smoking cessation treatment
}

\section{Paul Aveyard}

In this issue of Thorax Aubin and colleagues $^{1}$ report a further trial from the Varenicline Phase III Programme (see

Correspondence to: Dr Paul Aveyard, Division of Primary Care and Public Health, University of Birmingham, Birmingham B15 2T, UK; p.n.aveyard@bham.ac.uk page 717). The trials supporting registration contrasted bupropion with varenicline in a double placebo design. ${ }^{2}{ }^{3}$ This study examines the efficacy of varenicline against nicotine replacement therapy (NRT). In many countries, including the $\mathrm{UK}$, bupropion is rarely used and NRT is the predominant treatment offered in general practices and in specialist smoking cessation clinics. It is not practical to obtain placebo NRT, so this trial was of an open-label design. This publication follows a study by Stapleton et al ${ }^{4}$ with historical controls which showed that varenicline is superior to NRT in achieving abstinence and in reducing withdrawal phenomena such as urges to smoke and withdrawal symptoms.

Varenicline is licensed for smoking cessation around the world, but in the UK the National Institute for Health and Clinical Excellence (NICE) makes 
decisions about whether the benefits from licensed medications are worth the costs and therefore whether such interventions can be used in the National Health Service (NHS). NICE approved varenicline in 2007,5 but many local primary care organisations have limited the use of varenicline by making it a second-line treatment choice. The main reason given is the lack of trial data against the key competitor, NRT, but many have felt that the main reason was actually cost. Varenicline costs slightly more per treatment course than NRT or bupropion and considerably more than nortriptyline, an effective $e^{6}$ but unlicensed medication. The trial by Aubin and colleagues showing that varenicline is more effective than NRT and the trials of varenicline versus bupropion mean that varenicline must be considered a first-line choice in smoking cessation treatment. The recently published NICE guidance on smoking cessation affirmed this view, ${ }^{7}$ and it should be unnecessary for patients to have to try and to have failed with other medications before being offered varenicline in the NHS or in other health systems.

Although the statistically non-significant difference in continuous abstinence at 1 year in this trial can be considered as lack of evidence of long-term superiority, this would be clutching at straws. If we put together the data showing that varenicline is clearly more effective than bupropion $^{8}$ with data that bupropion and NRT are roughly equally effective, ${ }^{9}$ the data from this trial ${ }^{1}$ and from the other published head-to-head comparison with $\mathrm{NRT}^{4}$ and data from the UK stop smoking services, we see a consistent picture. Varenicline is clearly more effective. Furthermore, although it is somewhat more expensive per treatment course, the difference in cost is small and it is likely to be the most cost-effective medication available for smoking cessation. ${ }^{4}$ Thus, we are in the happy position of having a licensed medication approved for use by NICE that is more effective and more cost-effective than competitors. ${ }^{10}$ Should varenicline become the only medication to be used in smoking cessation?

In most areas of medicine the most effective and cost-effective medication would mean other choices were rarely used, but this is unlikely to be the case in smoking cessation. We need to remind ourselves that smoking is a behaviour largely explained by addiction, and one of the hallmarks of addiction is reinstatement after abstinence. Thus, in the study by Aubin et al ${ }^{1}$ which probably took place in the best clinics, only a quarter of smokers remained smoke-free for 1 year. One-third of these will probably resume smoking again in the next 2 years. ${ }^{11}$ Thus, in the best hands, 8 or 9 out of 10 treatment episodes end in failure. According to NICE, ${ }^{12}$ smoking cessation treatment remains "among the most costeffective of all healthcare interventions" because treatment is cheap and because the benefits of stopping smoking are so large in comparison to continuing. However, many failures occur early in treatment-nearly half those on varenicline had resumed smoking by the end of treatment in the trial by Aubin et al. ${ }^{1}$ In smoking cessation practice, then, we see a cadre of patients who have tried to stop many times and have, in effect, performed unblinded n-of-1 trials of all available smoking cessation medications. These patients are experts in which treatment works for them. Unlike many pharmacotherapies, patients can feel treatments for tobacco addiction working. Patients need to feel confident and content in their medication choice. In most conditions the main effort patients expend in treatment is remembering to take their drugs but, in smoking cessation, considerably more effort than this is usually required to achieve success. Confidence in the medication is probably important, and using patients' past experiences and preferences is more important in smoking cessation than in many other areas of medical practice.

One issue to highlight is the fact that the comparator treatment in the trial by Aubin et $a l^{1}$ was the nicotine patch. The patch is the most widely used NRT preparation in the $\mathrm{UK}^{13}$ and elsewhere because it is easy to use. However, in clinical practice it is common for patients to swap forms of NRT. Overall, there is insufficient evidence to suggest that one form is more effective than another, ${ }^{9}$ but the clinical impression is that allowing someone to renew their confidence by swapping from a choice they see as failing them to another can help.

An important finding from the trial by Aubin et $a l^{1}$ is that the urge to smoke and the occurrence of withdrawal symptoms were reduced with varenicline compared with NRT. Consensus guidelines on measuring and analysing urges and withdrawal suggest doing so primarily in abstinent participants only,$^{14}$ a practice not adopted by Aubin and colleagues. Data show that urges and withdrawal increase before relapse, ${ }^{15}$ so any arm that has a higher rate of relapse might have a higher level of urges and withdrawal and therefore these data might only show that resuming smoking was more likely on NRT. Although urges and withdrawal symptoms are associated with relapse, they are probably the cause of most relapses but it is difficult to demonstrate this conclusively. However, Stapleton analysed withdrawal only in largely abstinent smokers and found a similar pattern of results. ${ }^{4}$ It is likely, then, that the difference in withdrawal is real and that this is an important reason why varenicline is more effective.

It is relevant to ask why a partial nicotinic agonist such as varenicline can achieve greater suppression of nicotine withdrawal symptoms than a full agonist such as NRT. This is probably because the amount of nicotine absorbed from NRT is considerably less than from cigarettes for most smokers, ${ }^{16}$ and hence they suffer needless withdrawal. There is strong clinical evidence that $4 \mathrm{mg}$ gum is more effective than $2 \mathrm{mg}$ gum in more dependent smokers ( $85 \%$ increase in efficacy), and that adding two or more forms of NRT-most sensibly the patch plus a more rapid acting product (gum, nasal spray, inhalator or lozenge/microtab) - is more effective, increasing the chance of success by $35 \%$. ${ }^{9}$ The study by Stapleton et al showed that combination NRT was more effective than single form NRT with no evidence that varenicline was more effective than combination NRT. ${ }^{4}$ However, the study could not exclude a worthwhile difference in efficacy and the point estimate of effect favoured varenicline. The key message is that combining forms of NRT should be the norm in specialist smoking cessation clinics and considered for smokers who may have relapsed on NRT but are keen to use it again in other contexts.

Aubin and colleagues also report that reinforcement from cigarettes smoked while on medication was lower on varenicline than on NRT. However, these data are difficult to interpret. First, most people who sustained prolonged abstinence probably did not smoke at all after quit day if this population is typical. Nothing predicts relapse as strongly as early lapses. ${ }^{17}$ Thus, this analysis will be dominated by measurements taken at week 1, prior to quitting, when participants were using varenicline but participants randomised to NRT were on no medication. It is unclear whether reduced reinforcement from cigarettes smoked before quitting partly explains the efficacy of varenicline, but positive hedonic responses to cigarettes smoked after quitting are associated with a slight increase in relapse. ${ }^{18}$ These data suggest that 
treatment before quitting might be important. In fact, clinical data show that treatment of patients with a nicotine patch for 2 weeks before quitting is associated with a statistically significant increase in abstinence of $79 \%$ over postcessation use only. ${ }^{9}$ The effectiveness of pre-cessation use is best explained by reductions in reward from smoking weakening conditioned responses, but mediation analysis of the effect of pre-cessation NRT or varenicline on cigarette reward would be needed to confirm this. Whatever the mechanism, pre-cessation use of nicotine patch should be considered in some smokers, although further large trials are probably necessary before precessation use of NRT becomes the norm.

The results of the study by Aubin et al are to be welcomed because varenicline provides another option for smokers wanting to quit. We can now provide clearer statements to our patients that varenicline is the best single pharmacotherapy to assist an attempt to quit. Although varenicline is more effective than the current standard patch, there are good reasons to assume that NRT taken before quit day and in combination might be of similar efficacy. NRT may become even more effective if newer, higher dose, rapid release formulations become available. In my view, however, in varenicline we are close to an optimal treatment for nicotine dependence, but stopping smoking involves more than treating nicotine dependence. Most people who successfully reach the end of treatment will relapse to smoking. While taking longer courses of varenicline may prevent some relapse, lifetime varenicline is unlikely to be a major solution. Instead, we need to develop cognitive-behavioural interventions together with judicious use of various medications. Until then, varenicline is a welcome treatment option that is likely to prove popular with clinicians and patients.

Funding: PA is funded by the National Institute for Health Research (NIHR) but the views expressed are not necessarily those of the NIHR or the NHS.

Competing interests: PA has done consultancy work for the pharmaceutical and biotechnology industries that led to payments to him and his institution. This includes work for companies providing smoking cessation medication including McNeil, who manufacture NRT, and Pfizer, the makers of varenicline.

Thorax 2008:63:666-668. doi:10.1136/thx.2008.096081

\section{REFERENCES}

1. Aubin J-H, Bobak A, Britton JR, et al. Varenicline versus transdermal nicotine patch for smoking cessation: results from a randomised, open-label trial. Thorax 2008;63:717-24.

2. Jorenby DE, Hays JT, Rigotti NA, et al. Efficacy of varenicline, an alpha4beta2 nicotinic acetylcholine receptor partial agonist, vs placebo or sustained-release bupropion for smoking cessation: a randomized controlled trial. JAMA 2006;296:56-63.

3. Gonzales D, Rennard SI, Nides M, et al. Varenicline, an alpha4beta2 nicotinic acetylcholine receptor partia agonist, vs sustained-release bupropion and placebo for smoking cessation: a randomized controlled trial. JAMA 2006;296:47-55

4. Stapleton JA, Watson L, Spirling LI, et al. Varenicline in the routine treatment of tobacco dependence: a pre-post comparison with nicotine replacement therapy and an evaluation in those with mental illness. Addiction 2008;103:146-54

5. National Institute for Health and Clinical Excellence (NICE). Final appraisal determination for varenicline for smoking cessation. 2007. http:// guidance.nice.org.uk/page.aspx?0 $=431473$ (accessed 3 June 2008)

6. Hughes JR, Stead LF, Lancaster T. Antidepressants for smoking cessation. Cochrane Database Syst Rev 2007; (1):CD000031.

7. National Institute for Health and Clinical Excellence (NICE). Guidance on smoking cessation. London: NICE, 2008.

8. Cahill K, Stead LF, Lancaster T. Nicotine receptor partial agonists for smoking cessation. Cochrane Database Syst Rev 2007;(1):CD006103

9. Stead LF, Perera R, Bullen C, et al. Nicotine replacement therapy for smoking cessation. Cochrane Database Syst Rev 2008;(1):CD000146.

10. Stapleton JA. Is varenicline cost-effective enough to be funded by the NHS now? 2006. http://www.scsrn. org/policy_guidance/Narenicline_NHS_CE.pdf (accessed 16 Feb 2008).

11. Etter JF, Stapleton JA. Nicotine replacement therapy for long-term smoking cessation: a meta-analysis. Tob Control 2006;15:280-5.

12. National Institute for Health and Clinical Excellence. Guidance on the use of nicotine replacement therapy (NRT) and bupropion for smoking cessation. 2002. http://www.nice.org.uk/nicemedia/ pdf/NiceNRT39GUIDANCE.pdf laccessed 16 Feb 2008).

13. West R, DiMarino ME, Gitchell J, et al. Impact of UK policy initiatives on use of medicines to aid smoking cessation. Tob Control 2005;14:166-71.

14. Shiffman S, West RJ, Gilbert DG. Recommendation for the assessment of tobacco craving and withdrawal in smoking cessation trials. Tob Res 2004;6:599-614.

15. Piasecki TM. Relapse to smoking. Clin Psychol Rev 2006;296:196-215.

16. Johnstone E, Brown K, Saunders C, et al. Level of nicotine replacement during a quit-smoking attempt. Tob Res 2004:6:377-9.

17. Stapleton JA, Russell MA, Feyerabend C, et al. Dose effects and predictors of outcome in a randomized trial of transdermal nicotine patches in general practice. Addiction 1995;90:31-42.

18. Shiffman S, Ferguson SG, Gwaltney CJ. Immediate hedonic response to smoking lapses: relationship to smoking relapse, and effects of nicotine replacement therapy. Psychopharmacology (Berl) 2006; 184:608-18.

\section{Lung transplant and cystic fibrosis: what's new from the UK and France?}

\section{Peadar G Noone}

Although the median survival for patients with cystic fibrosis (CF) has improved steadily over the past several decades, many patients go on to develop respiratory failure from progressive lung disease, eventually requiring lung transplantation for extended survival. ${ }^{1}$ Although many years have elapsed since the first lung transplants were performed for CF, the

Correspondence to: Dr Peadar G Noone, Pulmonary Division, CB\# 7020, UNC Chapel Hill, NC 27599-7020, USA; pnoone@med.unc.edu field is not without controversy. ${ }^{2}$ The paper recently published by Liou et al is one recent example, suggesting that lung transplant for most children with CF under 18 years of age offers no survival advantage. ${ }^{2}$ The complex statistical methodology and conclusions have since been challenged and rebutted by several lung transplant experts. ${ }^{3-5}$ Controversial issues like this often reflect the shortage of randomised controlled trials for many aspects of lung transplant. Although there is much published material related to lung transplant, many protocols are based on retrospective data, or are rather centre or region specific. Although impure because of multiple confounding factors, such as small sample sizes, and varying surgical and medical protocols, these data do provide a reasonable template to formulate and update lung transplant protocols.

Two such datasets ${ }^{67}$ are published in this issue of Thorax (see pages 725 and 732). The first represents the experience of a single centre in the UK, accounting for a large proportion of lung transplants for CF in Britain, ${ }^{6}$ while the second paper from France addresses a difficult issuethat is, the risks of performing lung transplantation in patients infected with Burkholderia cepacia complex (BCC) organisms. The papers are complementary: the UK experience is a general report, excluding a detailed analysis of outcomes in patients with BCC (these 